\title{
Point-of-Care Ultrasound Use by Primary Care Physicians
}

\author{
Franklin Niblock, MD, MPH, Hoon Byun, DrPH, MA, and Yalda Jabbarpour, MD
}

Despite the proven benefits, less than $10 \%$ of physicians have adopted point-of-care ultrasound in primary care. Physician and practice characteristics, such as being a family physician and working in rural settings, increase the odds that a physician will adopt POCUS in their practice. ( $\mathrm{J}$ Am Board Fam Med 2021;34:859-860.)

Keywords: Family Physicians, Point-of-Care Systems, Primary Health Care, Rural Population, Ultrasonography

The American Academy of Family Physicians (AAFP) recently published a curriculum guideline calling point-of-care ultrasound (POCUS)—the largest advancement in bedside diagnosis since the advent of the stethoscope. ${ }^{1}$ POCUS refers to the use of portable ultrasound by a medical professional for diagnostic or procedural purposes. To date, POCUS in primary care has been shown to aid in the completion of procedures, ${ }^{2}$ lower costs, ${ }^{3}$ decrease emergency department visits, ${ }^{3}$ and reduce the need for further imaging. ${ }^{4}$ The 2018 American Board of Family Medicine Graduate Survey indicated that 14\% of graduates felt prepared to use musculoskeletal ultrasound, but no studies thus far have sought to understand the characteristics of who provides POCUS. ${ }^{5,6}$ Our objective was to understand predictors of POCUS use among primary care physicians (PCPs).

Through a retrospective claims-based approach, we analyzed select ultrasound examinations performed by PCPs and the performing physician's characteristics. Utilization data were gathered from the Medicare Part B Public Use Files from 2012 to

This article was externally peer reviewed.

Submitted 30 November 2020; revised 25 February 2021; accepted 11 March 2021.

From the Department of Family Medicine, University of Colorado School of Medicine, Aurora, CO (FN); The Robert Graham Center, Washington, DC (HB, YJ).

Funding: None.

Conflict of interest: None.

Corresponding author: Franklin Niblock, MD, MPH, Department of Family Medicine, University of Colorado School of Medicine, Mail Stop F496, 12631 East $17^{\text {th }}$ Avenue, Room 3510, Aurora, CO 80045, Phone: 704-6997088 (E-mail: franklin.niblock@CUanschutz.edu).

\section{See Related Article on Page 856.}

2017, and physician characteristics were supplemented by the 2018 American Medical Association Master File. PCPs were defined as those specializing in family medicine, internal medicine, general practice, and geriatric medicine. Common ultrasound examinations (diagnostic and procedural) performed by PCPs in the outpatient setting were identified by the AAFP's curriculum guideline on POCUS ${ }^{1}$ and matched to the most appropriate current procedural terminology, better known as CPT, code. Physicians billing for ultrasounds from 2012 to 2017 were classified as POCUS users. We assessed differences in POCUS use by physician demographics and practice characteristics. Logistic regression was used to determine the independent associations between these characteristics and the likelihood of POCUS use.

A total of 93,264 (9.3\%) primary care physicians were billed for an ultrasound from 2012 to 2017. Family physicians made up half $(52.2 \%)$ of outpatient providers billing for POCUS, followed closely by internists (43.7\%). General practitioners and geriatricians made up a small proportion of POCUS users. Statistically significant differences in POCUS use were seen across all reviewed characteristics. Being older, male, specializing in family medicine, practicing in the south, and practicing in a rural location were associated with increased odds of POCUS use [Figure 1].

We used a claims-based approach of Medicare Part B Public Use Files, limiting the capture of ultrasounds not billed for and those completed by a provider who billed for less than 11 ultrasounds within a year. Physicians are likely not billing for all POCUS exams they perform due in part to the added administrative burden of documenting and 
Figure 1. Adjusted Associations of Primary Care Physicians using Point-of-Care Ultrasound.

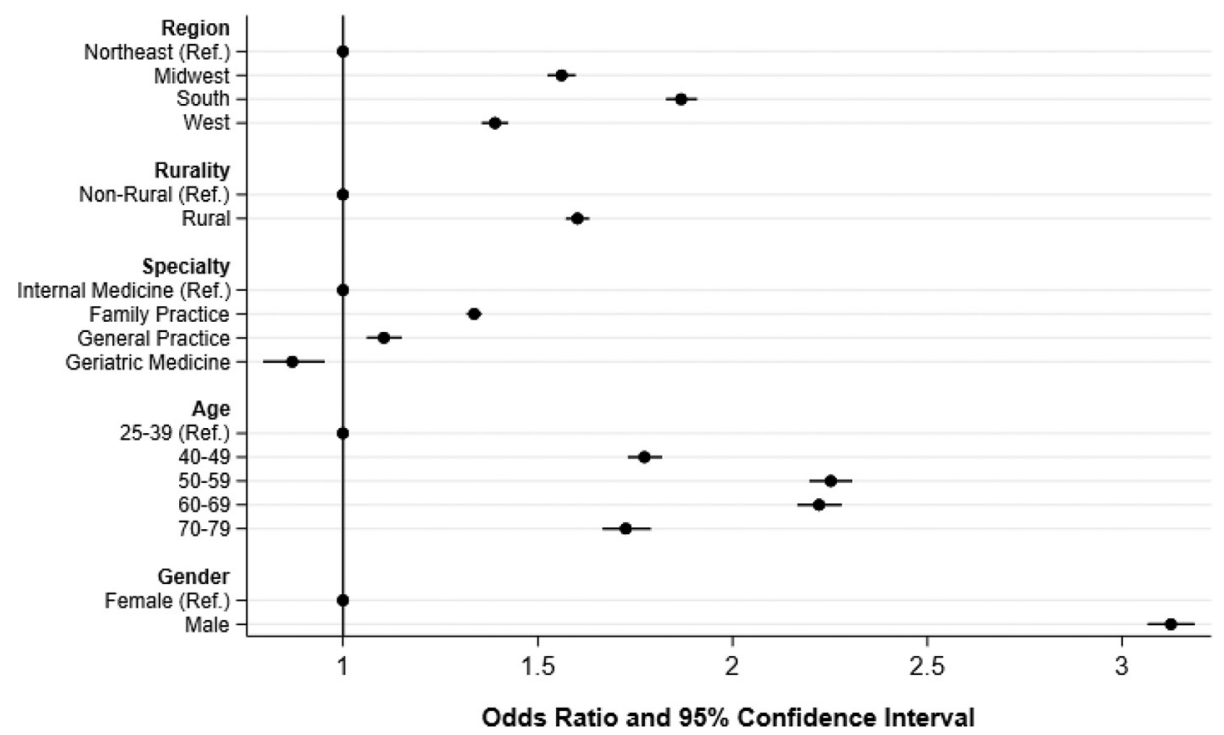

*Multivariate logistic regression including and controlling for all variables.

billing for exams. ${ }^{7}$ Physicians may perform POCUS without billing as they refine their skills in ultrasound or as they seek accreditation through their employer.

Although POCUS use is limited among PCPs, family physicians were more likely to perform POCUS than any other primary care specialty. Family physicians are primed to lead in the implementation of POCUS in primary care given their likely exposure to obstetric ultrasound, notably excluded in this analysis of Medicare patients. In addition, family physicians often practice as frontline physicians in resource-limited settings where POCUS may prove more useful. Future research should explore barriers to POCUS adoption to foster the spread of this valuable technology.

To see this article online, please go to: http://jabfm.org/content/ 34/4/859.full.

\section{References}

1. American Academy of Family Physicians (AAFP). Recommended curriculum guidelines for family medicine residents: point of care ultrasound. Available from: https://www.aafp.org/dam/AAFP/documents/ medical_education_residency/program_directors/ Reprint290D_POCUS.pdf. Published 2016. Accessed October 14, 2019.

2. Greenlund LJ, Merry SP, Thacher TD, Ward WJ. Primary care management of skin abscesses guided by ultrasound. Am J Med 2017;130:e19-e193.

3. Wordsworth S, Scott A. Ultrasound scanning by general practitioners: is it worthwhile? J Public Health Med 2002;24:88-94.

4. Evangelista A, Galuppo V, Méndez J, et al. Handheld cardiac ultrasound screening performed by family doctors with remote expert support interpretation. Heart 2016;102:376-82.

5. American Board of Family Medicine. 2018 Graduate Survey Report. Available at: https://www.theabfm. org/sites/default/files/2019-04/2018\%20National\% 20Graduate\%20Survey\%20Report_National\%20Only. pdf. Published 2018. Accessed May 12, 2020.

6. Sorensen B, Hunskaar S. Point-of-care ultrasound in primary care: a systematic review of generalist performed point-of-care ultrasound in unselected populations. Ultrasound J 2019;11:31.

7. Hughes D, Corrado MM, Mynatt I, Prats M, et al. Billing I-AIM: a novel framework for ultrasound billing. Ultrasound J 2020;12:8. 